\title{
GENERAL DISCUSSION
}

Bok: I think my message to Commission 33 will obviously be that we must be prepared to accept fainter absolute magnitudes for the RR Lyrae variables.

Oort: As Dr. Clube remarked, the discrepancy between the absolute magnitudes found from the reflection of the solar motion ('secular parallaxes') and that found from the peculiar motions ('dispersion') was also noted by others. From his extensive work on RR Lyrae-variable proper motions, Dr. van Herk found a difference in the same direction as that discussed by Clube, but somewhat less extreme. For the stars with low metal content the mean absolute magnitudes found by van Herk were about +0.4 and +0.9 , respectively, for the two kinds of data. I was also involved in the calculations; though we did not like the difference, we did not think it was exorbitant, being about twice its mean error. We certainly do not believe it can be ascribed to a considerable underestimate of the accidental errors of the proper motions, which were carefully discussed. There are, however, many uncertain data involved such as the determination of the true velocity dispersions along the three axes of the velocity ellipsoid.

I find it very difficult to believe that the secular parallaxes of the faint comparison stars such as used by van Herk could have been too small by the amount of the order of $0^{\prime \prime} .005$ pa suggested by Clube. It seems quite unlikely that stars at still quite moderate average distances from the galactic plane (about $200 \mathrm{pc}$ ) would show a much larger motion than the dynamically quite similar K-giants in our surroundings. (After the above discussion, Vasilevskis and Klemola went over their results for the motions of stars of the magnitudes concerned relative to faint galaxies. The difference between these preliminary results and Binnendijk's secular parallaxes which were used by van Herk averaged only between $0^{\prime \prime} .001$ and $0^{\prime \prime} .002$ pa, i.e., much smaller than the correction needed by Clube).

Adoption of a mean absolute magnitude of +1.3 for the RR Lyrae stars as proposed by Clube would in my opinion give very great difficulties in connection with the distance of the galactic center, $R_{0}$. This would then have to be reduced to $6.1 \mathrm{kpc}$ in the case of Arp's data on Baade's 'window', and even to about $4.7 \mathrm{kpc}$ in the case of Plaut's new surveys in fields at $b=-10^{\circ}$ and $+10^{\circ}$ and $l$ around $0^{\circ}$. Such small values are in contradiction with the value found from $21 \mathrm{~cm}$ observations (which give the product $A R_{0}$ ) and from distant $\mathrm{OB}$ stars at roughly the same distance from the center as the Sun. Both lead to values around $10 \mathrm{kpc}$.

Murray: Whatever the effects on the galactic distance scale, we must accept that there is an increasing amount of evidence that secular parallaxes of faint stars, out of the plane, are larger than had hitherto been thought. Until recently, no absolute proper motions for stars fainter than about the twelfth magnitude have been available for direct measurement of secular parallaxes, and we have had to rely on the statistics of velocity dispersions derived from local stars to measure mean parallaxes. Preliminary results of the Pulkovo proper motion programme, reported by Fatchikhin, indicate an increase in secular parallaxes, not only from the size of the motions but also in a shift of the solar apex corresponding to a higher negative $V$-component of motion.

Arp: What kind of absorption corrections did you apply to your apparent magnitudes to get absolute magnitudes?

Clube: The same corrections you used in your Kuiper Volume Chapter on Globular Clusters.

Arp: That would mean essentially cosecant law absorption corrections. It would be very important to check and see that the same kind of absorption corrections were used for your field RR Lyrae stars that I used in my computation of the distance to the center of the galaxy from the centroid of the globular clusters.

Another point which should be made is that if you imply your field RR Lyrae stars are the same as the globular cluster RR Lyrae stars, then your much lower absolute magnitude would imply a much greater age than presently assigned to the globular clusters or to the galaxy.

The most important point is what kind of star are you actually observing. If your low absolute magnitudes are correct, then you are probably dealing with a different kind of RR Lyrae star than is normally encountered in halo globular clusters. If my memory serves me, there was, some years ago, already an indication that field RR Lyrae stars were intrinsically fainter. That, of course, would not move the center of the galaxy closer than the currently calculated $10 \mathrm{kpc}$ or thereabouts.

Iben: In clusters which exhibit a main sequence turn off that can be related to the RR Lyrae variables directly above it in the H-R diagram, ages increase by about 3 billion yr per $0.25 \mathrm{mag}$. increase in the magnitude of RR Lyrae stars. The discussion by Dr. Clube refers to field RR Lyrae stars and not to 
variables in clusters. Hence, the suggested increase in magnitude cannot be directly transformed to cluster variables and then used to infer an increase in cluster ages.

Gliese: As Clube referred the proper motion of each RR Lyrae star to a frame of faint stars whose secular parallaxes he used, possible errors in the fundamental system cannot have any influence on his results.

Luyten: I have measured the 'motions' of some 30 quasars relative to some 8-10 comparison stars of the 16th and 17th mag. in each case. What one gets is the reverse of the mean motion of the comparison stars, and from these $I$ also found a pretty definite indication that the secular parallaxes of these very faint stars are larger than we had assumed before - in agreement with what Murray said.

Schwarzchild: Are the RR Lyrae stars in Plaut's low latitude field the same kind as the high latitude variables used by Clube?

Oort: The great majority of the variables in Plaut's field have periods in excess of 0.42 day and presumably belong to the low-metal-content category. 